Commentary on Maria and Andrea: Comparing Positive and Neqative Outcome Cases in an Online, Clinician-Guided, Self-Help Intervention for Panic Disorder; and

Daydreamer and Night Owl: Comparing Positive and Neqative Outcome Cases in an Online, Clinician-Guided, Self-Help Intervention for Social Anxiety Disorder

\title{
Case Studies of Clinician-Guided, Online Therapy: Towards a Fuller Understanding of How and Under What Conditions Such Therapy Works
}

\section{FRANZ CASPAR ${ }^{\mathrm{a}, \mathrm{b}}$}

${ }^{\text {a }}$ Clinical Psychology, University of Bern

${ }^{\mathrm{b}}$ Correspondence regarding this article should be sent to Franz Caspar, Clinical Psychology, Fabrikstrasse 8, 3012 Bern, Switzerland

Email: franz.caspar@psy.unibe.ch

\begin{abstract}
Ciuca, Berger, and Miclea (2017) and Schulz, Vincent, \& Berger (2017) each present a pair of process-focused, systematic case studies, one with good outcome and one with a poorer outcome. These cases were drawn from a randomized controlled trial (RCT) of an Online self-help therapy for panic disorder and social anxiety disorder, respectively. In both instances the cases were drawn from the clinician-guided arm of the RCT. This commentary reviews the kind of knowledge emerging from these pairs of case studies from a variety of perspectives, including: the practical advantages of online, clinician-guided treatment; client readiness for treatment; the role of the therapist alliance; the broad reach of online therapy; and the important complementary role that systematic case studies play in enhancing the knowledge that emerges from RCTs.
\end{abstract}

Key words: Online therapy; clinician-guided Online therapy; panic disorder; social anxiety disorder; case studies; clinical case studies; randomized controlled trials; therapeutic alliance

The systematic case studies by Ciuca, Berger, and Miclea (2017) and by Schulz, Vincent, and Berger (2017) are notable contributions to the growing body of literature in the field of Online treatments. While many randomized controlled trials (RCTs) show that Online interventions can work for a number of mental problems and disorders, case studies are still rare. Ciuca et al.'s and Schulz et al.'s systematic case studies, which each compare a good outcome and a poorer outcome case, offer several benefits and insights.

\section{PRACTICAL ADVANTAGES OF ONLINE TREATMENT AND THE NEED TO KNOW HOW THEY WORK}

Ciuca et al. (2017) and Schulz et al. (2017) present a convincing introduction into reasons why more varied and more efficient provision of therapy services is needed, including Online 
therapy. They also present convincing evidence supporting the use of Online therapy and make clear that today the question is less about whether change occurs in these forms of therapy, but rather more about how change occurs, including the mechanisms behind the change. For, as documented by Ciuca et al. and Schulz et al., there is strong proof of the practical advantages of Online therapy and its non-inferiority in outcome as compared to traditional face-to-face therapy. (Also it should be noted that co-author Thomas Berger brings to this research a very important background and context as a seasoned researcher on traditional therapies.)

A very important benefit of the two sets of case studies is the detailed description of the treatment process, which informs clinicians and researchers on the just-emphasized question of how these treatments concretely work. Most of the studies into Online treatments, including the two sets of case studies reviewed here, have investigated clinician-guided treatments. The two papers nicely demonstrate that clinician-guided Online therapy is different from other forms of Online therapy, such as psychotherapy via e-mail or chat in which the Internet is solely used as a communication medium. The main treatment component of the clinician-guided Online approach is a self-help program which is often based on cognitive behavioral therapy. Therapists are actively involved, although to a lesser degree than in traditional therapy. While a client works his or her way through the Online self-help program, a therapist assists and supports the client via real-time video sessions (e.g., Ciuca et al., 2017) or via e-mail (e.g., Schulz et al., 2017).

\section{CLIENT READINESS FOR TREATMENT}

The specific format of Online, clinician-guided treatment has an impact on how the Online interventions work, and it also requires specific skills and prerequisites of patients, such as motivation and intellectual abilities. As Ciuca et al. point out, Maria, the patient with a good outcome, was in an action-oriented stage according to the stages-of-change model (Prochaska, Norcross, \& DiClemente, 2013), whereas Andrea, the patient with the poorer outcome, seems to have been in an earlier stage of change at the beginning of treatment. In a related point, Schulz et al. note that both patients presented in their paper, Daydreamer and Night Owl, were highly educated, capable of introspection, and motivated to take part in the study. Therefore, an open question is whether these treatments also work for less educated and less motivated patients. It would be important to conduct more case studies to identify whether there are ways to adapt Online, clinician-guided interventions with clients who are less educated and/or less motivated.

With regard to the other reasons why this new treatment format works for certain patients but not others, both Ciuca et al.'s case study of Andrea and Shultz et al.'s case study of the Night Owl emphasize difficulties when patients present individual problems not covered in the standardized, disorder-specific self-help programs. Although new developments in the field of Online interventions include transdiagnostic and additive Online programs addressing comorbid symptoms, problems with Online treatments may arise when patients require individually tailored interventions and a therapeutic environment with nuanced and context-based therapist responsiveness (Kramer \& Stiles, 2015). 
The Ciuca et al. and Shultz et al. papers clearly show that there are differences between clients regarding the importance of tailoring the treatment and the importance of support and guidance by the therapist. For instance, in the Schulz et al. study, Daydreamer, the good-outcome client, needed less individualization and less support from the therapist than Night Owl, the poorer outcome client. In response to Night Owl's needs, the therapist tried to compensate the lack of individualization and fit of the treatment with more support and guidance, but this strategy was not successful.

\section{THE ROLE OF THE THERAPEUTIC ALLIANCE}

Research on clinician-guided self-help treatments also shows that there are differences between client groups regarding the importance of the therapeutic alliance. In a study in which our research team divided the participants of a guided self-help treatment into a group of (1) participants who used the possibility to write to their therapist frequently, and (2) participants who hardly ever wrote anything to their therapist. Whereas alliance scores in the second, "low contact group" were not predictive for outcome, a substantial correlation between the therapeutic alliance and outcome was found in the first, "intensive contact group” (Berger, Hohl, \& Caspar, 2009). Thus, research in the field of Online treatments may also inform research on traditional face-to-face psychotherapy, for example, on characteristics of clients that need or do not need a strong alliance.

In line with other research on the therapeutic alliance in Online treatments, the Ciuca et al. and Schultz et al. case studies show that although the contact with the therapist was limited, a good working alliance according to the patient's point of view can be established. Thus, it seems that the therapeutic alliance does not necessarily require direct face-to-face contact with a therapist. This finding is probably not surprising when one considers that, for example, we can also build a friendly relationship with a "pen pal” or an "email pal," or a love relationship through an internet dating service.

Criticism of Online treatments often emphasizes the deficits of written online communication, such as the lack of nonverbal and paraverbal cues. This criticism neglects the fact that the reduction of communication channels may also be compensated by the client feeling more open to verbalizing emotions without the close immediacy of another person, and this enhanced emotional expression may foster the alliance and/or may have a therapeutic effect in itself. Specifically, to this point, Suler (2014) presents anecdotal evidence that clients selfdisclose and express themselves more openly in online therapies.

In a related way, Suler (2004) has also summarized observations documenting that, in general,

people say and do things in cyberspace that they wouldn't ordinarily say and do in the faceto-face world. They loosen up, feel less restrained, and express themselves more openly. So pervasive is the phenomenon that a term has surfaced for it: the online disinhibition effect (p. 321). 
Suler proposes that the online disinhibition effect is created by the interaction of six factors, including "dissociative anonymity, invisibility, asynchronicity, solipsistic introjection, dissociative imagination, and minimization of authority” (p. 321; for more details, see Suler's article).

When defenders of face-to-face therapy argue that therapy without a personal, tangible relationship can't work, we should, first, remember that the correlations between quality of relationship and outcome are moderate, suggesting that outcome can be good in therapies with not-so-good relationships. Second, relationship-outcome correlations can only develop when there is variation, which means that bad or not-so-good relationships must exist. The absence of a relationship (see, however, the following paragraph) may be better for outcome than a bad relationship, which may overall compensate for the possible disadvantages of Online therapy and lead to overall similar effects.

An open question regarding the therapeutic alliance is whether alliance measures developed for the use in face-to-face therapies are also appropriate for use in Online treatments. It is very likely that established alliance measures do not capture specific aspects of Online relationships. This may especially be the case in therapies based on asynchronous written communication (e.g., Schulz et al., 2017), but also in therapies which include real-time video sessions (e.g., Ciuca et al., 2017). There is evidence showing that therapists and clients make adjustments in their behavior when conducting therapy via real-time video. For instance, Bischoff, Hollist, Smith, and Flack (2004) observed that therapists deliberately exaggerated nonverbal behavior in video-based therapy in order to compensate for the influences of the technology. In brief, technology seems to exert influences on human relationship-associated behavior. An important goal of future research should therefore be to better understand specific characteristics of the Online relationship.

\section{THE BROAD REACH OF ONLINE THERAPY}

The World Health Organization (WHO) has conducted mental health surveys in 28 countries and found that during their lifetime, close to half the global population reveals symptoms associated with a DSM-IV diagnosis (Kessler et al., 2009). Is it feasible to supply all these individuals with psychotherapy in a traditional sense? Kazdin and Blase (2011) make clear that there is no way that this could happen. A conclusion that emerges from their detailed arguments is that the great benefits of efficiency in Online therapy make it a very promising approach to complement traditional therapy.

The fact that the Ciuca et al. case studies and the Schulz et al. case studies were conducted in two contrasting countries-Romania and Switzerland, respectively-illustrate that Online interventions can be adapted to quite different geographical areas and countries.

Legal issues aside, it is very possible for therapists not only from but also in foreign countries to work with Online therapy users who are migrants or have no access to face-to-face psychotherapy for other reasons, such as war. For example, Knaevelsrud, Brand, Lange, Ruwaard, and Wagner (2015) have had extensive experience with Online therapy offered from 
Germany for PTSD patients in war-torn Iraq. These authors conducted an RCT, whose dramatic nature merits some details. Created in and managed by psychologists in Berlin, the therapy web site involved the screening, assessment, and delivery of Online therapy services to 79 such patients. A Dutch Online-based cognitive-behavior therapy manual was translated into Arabic and culturally adapted to the target population and research setting. The program consisted of 10 sessions, including exposure to trauma through writing assignments, cognitive restructuring, and social sharing. Comparison of outcomes on relevant standardized measures showed that PTSD symptoms were significantly reduced from baseline to posttreatment in the treatment group relative to a randomly assigned matched control group. It would be most valuable to see if the therapeutic power of Knaevelsrud et al.'s Online treatment could be enhanced by the addition of clinician guidance, and how this later approach could in turn be enhanced by the use of systematic case studies, as illustrated by the present case studies of Ciuca et al. and Schulz et al.

There are two particular arguments for the use of Online instead of face-to-face therapy for traumatized migrants. First of all, many of them come from cultures unfamiliar with traditional therapy, and shame and stigmatization is highly related to traumatic events such as rape. Second, traditional therapy typically requires interpreters. Normally these interpreters don't have clinical training to deal with possible secondary traumatization by the reports they hear and translate. This is a serious problem in Germany (e.g., Kilzilhan, 2016), with its massive number of refugees of whom a big percentage is traumatized by horrifying events; and this problem is much reduced with Online therapy by trained clinicians.

In sum, Ciuca et al.’s contrasting-outcome case studies of Maria and Andrea, and Shultz et al.'s contrasting-outcome case studies of Daydreamer and Night Owl-drawn from RCTsare a good illustration of the arguments by Fishman, Messer, Edwards, and Dattilio (2017) in favor of single case analyses as a crucial complement to RCTs, since "it is possible that important therapeutic processes have not been measured or are much too fine-grained or complex to be detected in existing studies" (Schulz et al., 2017, p. 219). Yes, this is why such case studies as commented on here are not just a gap-filler for missing, large-scale quantitative studies, but a true enrichment of research methodology and findings.

\section{REFERENCES}

Berger, T., Hohl, E., \& Caspar, F. (2009). Internet-based treatment for social phobia: A randomized controlled trial. Journal of Clinical Psychology, 65(10), 1021-1035.

Bischoff, R. J., Hollist, C. S., Smith, C. W., \& Flack, P. (2004). Addressing the mental health needs of the rural underserved: Findings from a multiple case study of a behavioral telehealth project. Contemporary Family Therapy: An International Journal, 26(2), 179198.

Ciuca, A.M., Berger, T., \& Miclea. M. (2017). Maria and Andrea: Comparing positive and negative outcome cases in an online, clinician-guided, self-help intervention for panic disorder. Pragmatic Case Studies in Psychotherapy, 13(3), Article 1, 173-216. Available: http://pcsp.libraries.rutgers.edu 
F. Caspar

Pragmatic Case Studies in Psychotherapy, http://pcsp.libraries.rutgers.edu

Volume 13, Module 3, Article 4, pp. 265-270, 11-11-17 [copyright by author]

Fishman, D.B., Messer, S.B., Edwards, D.J.A., \& Dattilio, F.M. (2017)(Eds.). Case studies within psychotherapy trials: Integrating qualitative and quantitative methods. Oxford: Oxford University Press.

Kazdin, A. E., \& Blase, S. L. (2011). Rebooting psychotherapy research and practice to reduce the burden of mental illness. Perspectives on Psychological Science, 6(1), 21-37.

Kessler R.C., Aguilar-Gaxiola S., Alonso J., Chatterji S., Lee S., Ormel J., (2009). The global burden of mental disorders: An update from the WHO World Mental Health (WMH) Surveys. Epidemiologia e Psichiatria Sociale, 18, 23-33.

Kilzilhan, J. (2016). Handbuch kriegstraumatisierter Frauen. Berlin: VBW Verlag.

Knaevelsrud, C., Brand, J., Lange, A., Ruwaard, J., \& Wagner, B. (2015). Web-based psychotherapy for posttraumatic stress disorder in war-traumatized Arab patients: Randomized controlled trial. Journal of Medical Internet Research, 17(3), e71.

Kramer, U., \& Stiles, W.B. (2015) The responsiveness problem in psychotherapy: A review of proposed solutions. Clinical Psychology: Science and Practice, 22, 277-295.

Prochaska, J. O., Norcross, J. C., \& DiClemente, C. C. (2013). Applying the stages of change [online]. Psychotherapy in Australia, 19(2), 10-15.

Schulz, A., Vincent, A., \& Berger, T. (2017). Daydreamer and Night Owl: Comparing positive and negative outcome cases in an online, clinician-guided, self-help intervention for social anxiety disorder. Pragmatic Case Studies in Psychotherapy, 13(3), Article 2, 217252. Available: http://pcsp.libraries.rutgers.edu

Suler, J. (2004). The online disinhibition effect. Cyberpsychology \& Behavior, 7(3), 321-326. 